\title{
Cooperative Communications with Relay-Selection: When to Cooperate and Whom to Cooperate With?
}

\author{
Ahmed S. Ibrahim, Student Member, IEEE, Ahmed K. Sadek, Member, IEEE, Weifeng Su, Member, IEEE, \\ and K. J. Ray Liu, Fellow, IEEE
}

\begin{abstract}
In this paper, we propose a new cooperative communication protocol, which achieves higher bandwidth efficiency while guaranteeing the same diversity order as that of the conventional cooperative schemes. The proposed scheme considers relay selection via the available partial channel state information (CSI) at the source and the relays. In particular, we discuss the multi-node decode-and-forward cooperative scenarios, where arbitrary $N$ relays are available. The source determines when it needs to cooperate with one relay only, and which relay to cooperate with in case of cooperation, i.e., "When to cooperate?" and "Whom to cooperate with?". An optimal relay is the one which has the maximum instantaneous scaled harmonic mean function of its source-relay and relay-destination channel gains. For the symmetric scenario, we derive an approximate expression of the bandwidth efficiency and obtain an upper bound on the symbol error rate (SER) performance. We show that full diversity is guaranteed and that a significant increase of the bandwidth efficiency is achieved. Moreover, we present the tradeoff between the achievable bandwidth efficiency and the corresponding SER. Finally, the obtained analytical results are verified through computer simulations.
\end{abstract}

Index Terms-Cooperative diversity, decode-and-forward cooperative protocol, multi-node wireless relay networks, optimal relay selection.

\section{INTRODUCTION}

M ULTIPATH fading is one of the major obstacles for the next generation wireless networks, which require high bandwidth efficiency services. Time, frequency, and spatial diversity techniques are used to mitigate the fading phenomenon. Recently, cooperative communications for wireless networks have gained much interest due to its ability to mitigate fading in wireless networks through achieving spatial diversity, while resolving the difficulties of installing multiple antennas on small communication terminals. In cooperative communication, a number of relay nodes are assigned to help a source in forwarding its information to its destination, hence forming a virtual antenna array.

Various cooperative diversity protocols were proposed and analyzed in [1]-[12]. In [2], Laneman et al. described various

Manuscript received February 12, 2007; revised October 16, 2007; accepted December 11, 2007. The associate editor coordinating the review of this paper and approving it for publication was T. Hou.

A. S. Ibrahim and K. J. R. Liu are with the Department of Electrical and Computer Engineering, University of Maryland, College Park, MD, 20742 USA (e-mail: \{asalah, kjrliu\}@umd.edu).

A. K. Sadek is with Corporate Research and Development, Qualcomm Incorporated, San Diego, CA, 92121 USA (e-mail: asadek@qualcomm.com).

W. Su is with the Department of Electrical Engineering, State University of New York at Buffalo, Buffalo, NY, 14260 USA (e-mail: weifeng@eng.buffalo.edu).

Digital Object Identifier 10.1109/TWC.2008.070176. techniques of cooperative communication, such as decodeand-forward, amplify-and-forward, selection relaying, and incremental relaying. It was shown that the first two schemes achieve bandwidth efficiency equal to $1 / 2$ symbols per channel use (SPCU), while the other two schemes achieve higher bandwidth efficiency. In [3], a distributed space-time coded (STC) cooperative scheme was proposed, where the relays decode the received symbols from the source and utilize a distributed space-time code. Su et al. derived symbol error rate (SER) for single-relay decode-and-forward and amplify-andforward cooperative techniques in [4] and [5], respectively. In [6], Sadek et al. provided SER performance analysis for the decode-and-forward multi-node schemes.

There are various protocols proposed to choose the best relay among a collection of available relays in the literature. In [7], the authors proposed to choose the best relay depending on its geographic position, based on the geographic random forwarding (GeRaF) protocol proposed in [8] and [9]. In GeRaF, the source broadcasts its data to a collection of nodes and the node that is closest to the destination is chosen in a distributed manner to forward the source's data to the destination. In [10], the authors considered a best-select relay scheme in which only the relay, which has received the transmitted data from the source correctly and has the highest mean signal-to-noise ratio (SNR) to the destination node, is chosen to forward the source's data. In [11], a relay-selection scheme for singlerelay decode-and-forward cooperative systems was proposed. In this scheme, the source decides whether to employ the relay in forwarding its information or not, depending on the instantaneous values of the source-destination and source-relay channels gain.

In this paper, we propose a cooperative protocol based on relay-selection technique using the availability of the partial channel state information (CSI) at the source and the relays. Partial CSI expresses the instantaneous channel gain without the phase component. The main objective of this scheme is to achieve higher bandwidth efficiency while guaranteeing the same diversity order as that of the conventional cooperative scheme. We consider a multi-relay scenario, where arbitrary $N$ relays are available and we address two main questions: "When to cooperate?" and "Whom to cooperate with?". The rationale behind this protocol is that there is no need for the relay to forward the source's information if the direct link, between the source and destination, is of high quality. In addition, the source picks only one relay to cooperate with in case it needs help. This optimal relay is the one which 
has the maximum instantaneous value of the relay's metric, which is a modified version of the harmonic mean function of its source-relay and relay-destination instantaneous channel gains, among the $N$ relays.

The source decides when to cooperate by taking the ratio between the source-destination channel gain and the optimal relay's metric and comparing it to a threshold, which is referred to as cooperation threshold. If this ratio is greater than or equal to the cooperation threshold, then the source sends its information to the destination directly without the need for any relay. Otherwise, the source employs the optimal relay in forwarding its information to the destination as in the conventional cooperative scheme. For the symmetric scenario, we provide an approximate expression of the bandwidth efficiency and an upper bound on the SER performance. In addition, we prove that full diversity order is guaranteed and obtain the optimum power allocation which minimizes the SER performance. Finally, we provide tradeoff curves between the bandwidth efficiency and the SER, which are utilized to determine the cooperation threshold.

The rest of this paper is organized as follows. In Section II, we introduce the conventional decode-and-forward cooperative scenario, which leads to the motivation behind choosing an appropriate metric to indicate the relay's ability to help. Furthermore, we introduce the multi-node relay-selection decode-and-forward cooperative scenario. In Section III, the performance analysis of the proposed scheme is presented with formulas for the approximate bandwidth efficiency and the SER upper bound. Section IV presents a solution to the optimum power allocation problem. Moreover, bandwidth efficiency-SER tradeoff curves for different SNR are also shown in this Section. We present some simulation results in Section V, which verify the analytical results. Finally, Section VI concludes the paper.

\section{Motivation And Proposed Relay-Selection PROTOCOL}

In this section, we present the system model of the conventional single-relay decode-and-forward cooperative scenario along with the SER results obtained in [4]. This helps in illustrating the motivation behind choosing a modified harmonic mean function of the source-relay and relay-destination channels gain as an appropriate metric to represent the relay's ability to help the source. Finally, we introduce the proposed multi-node relay-selection decode-and-forward cooperative scenario.

\section{A. Conventional Single-Relay Decode-and-Forward Coopera- tive Scenario}

The communication system of a conventional single-relay decode-and-forward cooperative scheme is shown in Fig. 1. It consists of a source, $s$, its destination, $d$, and a relay, $r$. The transmission protocol requires two consecutive phases as follows. In the first phase, the source broadcasts its information to the relay and the destination. The received symbols at the destination and relay can be modeled as $y_{s, d}=\sqrt{P_{1}} h_{s, d} x+\eta_{s, d}$ and $y_{s, r}=\sqrt{P_{1}} h_{s, r} x+\eta_{s, r}$, where $P_{1}$ is the source transmitted power, $x$ is the transmitted

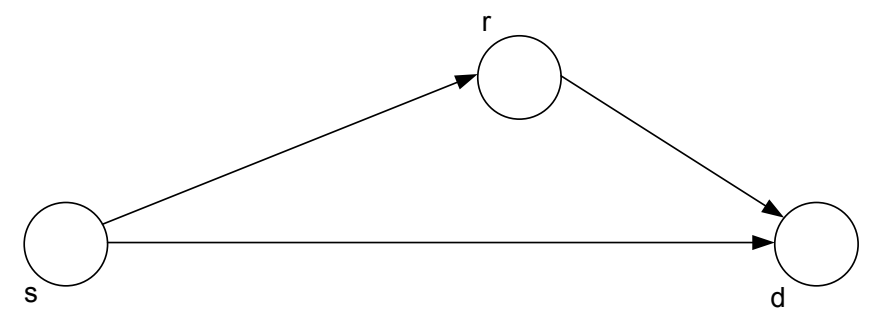

Fig. 1. Single-relay cooperative communication system.

information symbol, and $\eta_{s, d}$ and $\eta_{s, r}$ are additive noises. Also, $h_{s, d}$ and $h_{s, r}$ are the source-destination and source-relay channel gains, respectively.

The relay decides whether to forward the received information or not according to the quality of the received signal. If the relay decodes the received symbol correctly, then it forwards the decoded symbol to the destination in the second phase, otherwise it remains idle. The received symbol at the destination from the relay is written as $y_{r, d}=\sqrt{\tilde{P}_{2}} h_{r, d} x+\eta_{r, d}$, where $\tilde{P}_{2}=P_{2}$ if the relay decodes the symbol correctly, otherwise $\tilde{P}_{2}=0, \eta_{r, d}$ is an additive noise, and $h_{r, d}$ is the relay-destination channel coefficient. The destination applies maximal-ratio combining (MRC) [13] for the received signals from the source and the relay. The output of the MRC can be written as $y=\frac{\sqrt{P_{1}} h_{s, d}^{*}}{N_{0}} y_{s, d}+\frac{\sqrt{\tilde{P}_{2}} h_{r, d}^{*}}{N_{0}} y_{r, d}$. The channel coefficients $h_{s, d}, h_{s, r}$, and $h_{r, d}$ are modeled as zero-mean complex Gaussian random variables with variances $\delta_{s, d}^{2}, \delta_{s, r}^{2}$, and $\delta_{r, d}^{2}$, respectively. The noise terms $\eta_{s, d}, \eta_{s, r}$, and $\eta_{r, d}$ are modeled as zero-mean complex Gaussian random variables with variance $N_{0}$.

It has been shown in [4] that the SER for M-PSK signalling can be upper bounded as

$$
\operatorname{Pr}(e) \leq \frac{N_{0}^{2}}{b^{2}} \cdot \frac{A^{2} P_{2} \delta_{r, d}^{2}+B P_{1} \delta_{s, r}^{2}}{P_{1}^{2} P_{2} \delta_{s, d}^{2} \delta_{s, r}^{2} \delta_{r, d}^{2}},
$$

where $b=\sin ^{2}(\pi / M)$,

$$
A=\frac{1}{\pi} \int_{0}^{\frac{(M-1) \pi}{M}} \sin ^{2} \theta d \theta=\frac{M-1}{2 M}+\frac{\sin \left(\frac{2 \pi}{M}\right)}{4 \pi},
$$

and

$$
\begin{aligned}
& B= \\
& \frac{1}{\pi} \int_{0}^{\frac{(M-1) \pi}{M}} \sin ^{4} \theta d \theta=\frac{3(M-1)}{8 M}+\frac{\sin \left(\frac{2 \pi}{M}\right)}{4 \pi}-\frac{\sin \left(\frac{4 \pi}{M}\right)}{32 \pi} .
\end{aligned}
$$

Moreover, it was shown in [4] that the SER upper bound in (1) is tight at high enough SNR.

\section{B. Relay-Selection Criterion}

In this subsection, we introduce a relay-selection criterion from the SER expression in (1). Let $\gamma \triangleq \frac{P}{N_{0}}$ denote the signalto-noise ratio (SNR), where $P=P_{1}+P_{2}$ is the total power. Hence, (1) can be written as

$$
\operatorname{Pr}(e) \leq(C G \gamma)^{-2},
$$


where $C G$ denotes the coding gain and it is equal to

$$
C G=\sqrt{b^{2} \delta_{s, d}^{2}\left(\frac{\delta_{s, r}^{2} \delta_{r, d}^{2}}{q_{1} \delta_{r, d}^{2}+q_{2} \delta_{s, r}^{2}}\right)},
$$

where

$$
q_{1}=\frac{A^{2}}{r^{2}}, \quad q_{2}=\frac{B}{r(1-r)},
$$

and $r \triangleq \frac{P_{1}}{P}$ is referred to as power ratio. The diversity order is defined as $d=-\lim _{S N R \longrightarrow \infty} \log (\operatorname{Pr}(e)) / \log (S N R)$. So, in (3) the tight SER upper bound expression has diversity order 2. Hence, the actual SER of the system has diversity order 2 as well. Generally, diversity of order $K$ means that there are $K$ independent paths from the source to the destination.

We note that maximizing the coding gain in (4) results in minimizing the SER in (3). By investigating the $C G$ in (4), we can see that the term $m \triangleq \frac{\delta_{s, r}^{2} \delta_{r, d}^{2}}{q_{1} \delta_{r, d}^{2}+q_{2} \delta_{s, r}^{2}}$ is the only term that depends on the relay channels (source-relay and relaydestination). Thus, if $N$ relays are available and we need to choose one relay only, we will choose the relay with maximum $m$. By doing so, the multi-relay scheme becomes a single-relay scheme that uses the best relay during the whole transmission time, because the metric $m$ depends on the average channel gains. Thus, the SER of this scheme is upper bounded as in (3). In other words, this scheme achieves diversity order 2 and not $N+1$ as we aim to achieve.

The main reason for this system not to achieve full diversity order is that one relay is chosen at the beginning of the transmission and it is used until the end of the transmission. If we can have the chance to choose the best relay at each time instant and utilize that relay only, then full diversity order can be achieved. Intuitively, this can be explained as follows. In order for the transmitted data to be lost, the direct path and the best-relay path have to be in deep fade. Consequently, all the other $N-1$ relay-dependent paths have to be in deep fade as the best-relay path is in deep fade. Thus a total of $N+1$ paths must be in deep fade to lose the transmitted signal. This corresponds to full diversity of order $N+1$ as explained above.

Since the average metric $m$ cannot achieve the full diversity order, we propose to replace the source-relay and relaydestination channel gains by their corresponding instantaneous channel gains, i.e., $\delta_{s, r}^{2}$ and $\delta_{r, d}^{2}$ are replaced by $\left|h_{s, r}\right|^{2}$ and $\left|h_{r, d}\right|^{2}$, respectively. By doing so, we are combining what we have concluded from the single-relay SER (i.e., the relay average metric $m$ ) along with the instantaneous information that can achieve full diversity order if utilized properly. Thus, the instantaneous relay metric can be written as $m^{\prime}=\frac{\left|h_{s, r}\right|^{2}\left|h_{r, d}\right|^{2}}{q_{1}\left|h_{r, d}\right|^{2}+q_{2}\left|h_{s, r}\right|^{2}}$. Finally, the metric $m^{\prime}$ is scaled to be in a standard harmonic mean function as $2 q_{1} q_{2} \mathrm{~m}^{\prime}$. Therefore, we propose the relay's metric $\beta_{m}$, which is given by

$$
\beta_{m}=\mu_{H}\left(q_{1} \beta_{r, d}, q_{2} \beta_{s, r}\right) \triangleq \frac{2 q_{1} q_{2} \beta_{s, r} \beta_{r, d}}{q_{1} \beta_{r, d}+q_{2} \beta_{s, r}},
$$

where $\beta_{s, r}=\left|h_{s, r}\right|^{2}, \beta_{r, d}=\left|h_{r, d}\right|^{2}$, and $\mu_{H}(.,$.$) denotes the$ standard harmonic mean function. The relay's metric $\beta_{m}$ (6) gives an instantaneous indication about the relay's ability to cooperate with the source.

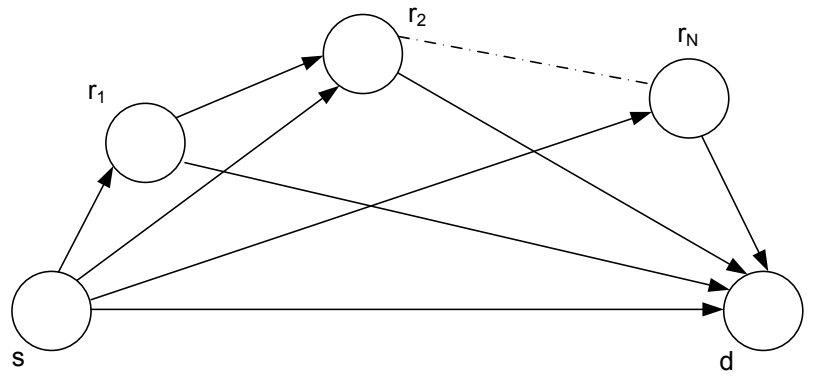

Fig. 2. Multi-node cooperative communication system.

\section{Proposed Relay-Selection Protocol}

The communication system of a conventional multi-node decode-and-forward cooperative scheme is shown in Fig. 2. The conventional multi-node decode-and-forward scheme is implemented in $N+1$ time slots (phases) as follows. In the first phase, the source broadcasts its data, which is received by the destination as well as the $N$ relays. The first relay decodes what it has received from the source and checks if it has received the data correctly. If it has received the data correctly, it re-encodes the data to be broadcasted in the second phase. Otherwise, it remains idle. Generally in the $i$-th phase, the $(i-1)$-th relay combines the signals coming from all the previous relays and the source, re-transmits the data if it has decoded correctly, and remains idle otherwise. Based on that model, $N+1$ time slots are needed to send 1 symbol. Thus, the bandwidth efficiency is $1 /(N+1)$ symbols per channel use (SPCU).

The basic idea of the proposed multi-node relay-selection cooperative scenario depends on selecting one relay among the $N$ relays to cooperate with the source, if it needs cooperation. There are two main questions to be answered. The first question is how to determine the optimal relay to cooperate with, in case of cooperation. The answer comes from the motivation described earlier. The modified harmonic mean function of the source-relay and relay-destination channel gains is an appropriate measure on how much help a relay can offer. Thus, the optimal relay is the relay with the maximum modified harmonic mean function of its sourcerelay and relay-destination channel gains among all the $N$ relays. With this optimal relay being decided, the system consists of the source, the destination, and the optimal relay, which is similar to the single-relay system shown in Fig. 1. The second question is how the source determines whether to cooperate with this optimal relay or not, and its answer is explained in the sequel while explaining the transmission protocol.

Let the metric for each relay be defined as the modified harmonic mean function of its source-relay and relay-destination channel gains as

$$
\begin{aligned}
\beta_{i} & =\mu_{H}\left(q_{1} \beta_{r_{i}, d}, q_{2} \beta_{s, r_{i}}\right) \\
& =\frac{2 q_{1} q_{2} \beta_{r_{i}, d} \beta_{s, r_{i}}}{q_{1} \beta_{r_{i}, d}+q_{2} \beta_{s, r_{i}}}, \text { for } i=1,2, \cdots, N .
\end{aligned}
$$

Consequently the optimum relay will have a metric, which is 
equal to

$$
\beta_{\max }=\max \left\{\beta_{1}, \beta_{2}, \ldots, \beta_{N}\right\} .
$$

The transmission protocol can be described as follows. In the first phase, the source computes the ratio $\beta_{s, d} / \beta_{\max }$ and compares it to the cooperation threshold $\alpha$. If $\frac{\beta_{s, d}}{\beta_{m a x}} \geq \alpha$, then the source decides to use direct transmission only. This mode is referred to as the direct-transmission mode. Let $\phi=\left\{\beta_{s, d} \geq \alpha \beta_{\max }\right\}$ be the event of direct transmission. The received symbol at the destination can then be modeled as

$$
y_{s, d}^{\phi}=\sqrt{P} h_{s, d} x+\eta_{s, d},
$$

where $P$ is the total transmitted power, $x$ is the transmitted symbol with unit average energy, $h_{s, d}$ is the source-destination channel coefficient, and $\eta_{s, d}$ is an additive noise.

On the other hand, if $\frac{\beta_{s, d}}{\beta_{\max }}<\alpha$, then the source employs the optimal relay $r$ to transmit its information as in the conventional single-relay decode-and-forward cooperative protocol [4]. This mode is denoted by relay-cooperation mode and can be described as follows. In the first phase, the source broadcasts its symbol to both the optimal relay and the destination. The received symbols at the destination and the optimal relay can be modeled as

$y_{s, d}^{\phi^{c}}=\sqrt{P_{1}} h_{s, d} x+\eta_{s, d}, \quad y_{s, r}^{\phi^{c}}=\sqrt{P_{1}} h_{s, r} x+\eta_{s, r}$,

respectively, where $P_{1}$ is the source transmitted power, $h_{s, r}$ is the source-relay channel coefficient, $\eta_{s, r}$ is an additive noise, and $\phi^{c}$ denotes the complement of the event $\phi$. The optimal relay decodes the received symbol and re-transmits the decoded symbol if correctly decoded in the second phase, otherwise it remains idle. The received symbol at the destination is written as

$$
y_{r, d}^{\phi^{c}}=\sqrt{\tilde{P}_{2}} h_{r, d} x+\eta_{r, d},
$$

where $\tilde{P}_{2}=P_{2}$ if the relay decodes the symbol correctly, otherwise $\tilde{P}_{2}=0, h_{r, d}$ is the relay-destination channel coefficient, and $\eta_{r, d}$ is an additive noise. Power is distributed between the source and the optimal relay subject to the power constraint $P_{1}+P_{2}=P$. We note that the optimal relay decides whether to forward the received information or not according to the quality of the received signal. For mathematical tractability, we assume that the relay can tell whether the information is decoded correctly or not ${ }^{1}$.

We assume that the channels are reciprocal as in the Time Division Duplex (TDD) mode, hence each relay knows its source-relay and relay-destination channel gains and calculates their harmonic mean function. Then, each relay sends this metric to the source through a feedback channel. Furthermore, we assume that the source knows its source-destination channel gain. Thus, the source uses its source-destination channel gain and the maximum metric of the relays, to determine whether to cooperate with one relay only or not. Finally, the source sends a control signal to the destination and the relays to indicate its decision and the optimal relay it is going to cooperate with, in case of cooperation. This procedure is repeated every time

\footnotetext{
${ }^{1}$ Practically, this can be done at the relay by applying a simple SNR threshold on the received data. Although, it can lead to some error propagation, but for practical ranges of operating SNR, the event of error propagation can be assumed negligible.
}

the channel gains vary. We assume that the channel gains vary slowly so that the overhead resulting from sending the relays' metrics is negligible. We should note here that the source and the relays are not required to know the phase information of their channels. Hence, only partial CSI is needed for this proposed algorithm.

Flat quasi-static fading channels are considered, hence the channel coefficients are assumed to be constant during a complete frame, and can vary from a frame to another independently. Rayleigh fading channel model is considered for the channel between each two nodes. Let $h_{i, j}$ be a generic channel coefficient representing the channel between any two nodes. $h_{i, j}$ is modeled as zero-mean complex Gaussian random variables with variance $\delta_{i, j}^{2}$. Thus, the channel gain $\left|h_{i, j}\right|$ is modeled a Rayleigh random variable. Furthermore the channel gain squared $\left|h_{i, j}\right|^{2}$ is modeled as an exponential random variable with mean $\delta_{i, j}^{2}$, i.e., $p\left(\left|h_{i, j}\right|^{2}\right)=1 / \delta_{i, j}^{2} \exp \left(-\left|h_{i, j}\right|^{2} / \delta_{i, j}^{2}\right)$ is the probability density function (PDF) of $\left|h_{i, j}\right|^{2}$. The noise terms, $\eta_{s, d}, \eta_{s, r}$, and $\eta_{r, d}$, are modeled as zero-mean, complex Gaussian random variables with equal variance $N_{0}$.

\section{Performance Analysis}

In this section, first we calculate the probability of the direct-transmission and relay-cooperation modes for the multinode relay-selection decode-and-forward cooperative scenario. Then, they are used to obtain an approximate expression of the bandwidth efficiency and an upper bound on the SER performance.

\section{A. Average Bandwidth Efficiency Analysis}

We derive the average achievable bandwidth efficiency as follows. The cumulative distribution function (CDF) of $\beta_{i}$ for $i=1,2, \cdots, N$, denoted by $P_{\beta_{i}}($.$) , can be written as given$ in $[14]$ as

$$
P_{\beta_{i}}\left(\beta_{i}\right)=1-\frac{\beta_{i}}{t_{1, i}} \exp \left(-\frac{t_{2, i}}{2} \beta_{i}\right) K_{1}\left(\frac{\beta_{i}}{t_{1, i}}\right),
$$

where $t_{1, i}=\sqrt{q_{1} q_{2} \delta_{s, r_{i}}^{2} \delta_{r_{i}, d}^{2}}, t_{2, i}=\frac{1}{q_{2} \delta_{s, r_{i}}^{2}}+\frac{1}{q_{1} \delta_{r_{i}, d}^{2}}$, and $K_{1}(x)$ is first-order modified Bessel functions of the second kind, defined in [[16], (9.6.22)]. The CDF of $\beta_{\max }$ can be written as

$$
P_{\beta_{\max }}(\beta)=\operatorname{Pr}\left(\beta_{1} \leq \beta, \ldots, \beta_{N} \leq \beta\right)=\prod_{i=1}^{N} P_{\beta_{i}}(\beta),
$$

and the PDF of $\beta_{\max }$ is written as

$$
\begin{aligned}
p_{\beta_{\max }}(\beta) & =\frac{\partial P_{\beta_{\max }}(\beta)}{\partial \beta} \\
& \approx \sum_{j=1}^{N} p_{\beta_{j}}(\beta)\left(\prod_{i=1, i \neq j}^{N}\left(1-\exp \left(-\frac{t_{2, i}}{2} \beta\right)\right)\right),
\end{aligned}
$$

where $p_{\beta_{j}}($.$) is the PDF of \beta_{j}$. In (14), we approximated $K_{1}($. as given in [[16], (9.6.9)] by

$$
K_{1}(x) \approx \frac{1}{x} .
$$




$$
\begin{gathered}
P_{\beta_{\text {max }}}(\beta)=\left(1-\frac{\beta}{t_{1}} \exp \left(-\frac{t_{2}}{2} \beta\right) K_{1}\left(\frac{\beta}{t_{1}}\right)\right)^{N} \\
\text { and } p_{\beta_{\text {max }}}(\beta)=N\left(1-\frac{\beta}{t_{1}} \exp \left(-\frac{t_{2}}{2} \beta\right) K_{1}\left(\frac{\beta}{t_{1}}\right)\right)^{N-1} p_{\beta_{m}}(\beta) \\
p_{\beta_{m}}\left(\beta_{m}\right)=\frac{\beta_{m}}{2 t_{1}^{2}} \exp \left(-\frac{t_{2}}{2} \beta_{m}\right)\left(t_{1} t_{2} K_{1}\left(\frac{\beta_{m}}{t_{1}}\right)+2 K_{0}\left(\frac{\beta_{m}}{t_{1}}\right)\right) U\left(\beta_{m}\right) \\
\operatorname{Pr}(\phi)=\operatorname{Pr}_{\left(\beta_{s, d} \geq \alpha \beta_{m a x}\right)=\int_{0}^{\infty} P_{\beta_{m a x}}\left(\frac{\beta_{s, d}}{\alpha}\right) p_{\beta_{s, d}}\left(\beta_{s, d}\right) d \beta_{s, d}} \\
=\sum_{n=0}^{N}\left(\begin{array}{l}
N \\
n
\end{array}\right)(-1)^{n} \frac{1}{\left(\alpha t_{1}\right)^{n} \delta_{s, d}^{2}} \int_{0}^{\infty} \beta_{s, d}^{n} \exp \left(-\left(\frac{1}{\delta_{s, d}^{2}}+\frac{t_{2} n}{2 \alpha}\right) \beta_{s, d}\right)\left(K_{1}\left(\frac{\beta_{s, d}}{\alpha t_{1}}\right)\right)^{n} d \beta_{s, d} . \\
\sum_{n=0}^{N}\left(\begin{array}{l}
N \\
n
\end{array}\right)(-1)^{n} \frac{2 \alpha}{2 \alpha+t_{2} \delta_{s, d}^{2} n}
\end{gathered}
$$

The expression in (14) is complex. For mathematical simplicity, we consider the symmetric scenario where all the relays have the same source-relay and relay-destination channel variances, i.e., $\delta_{s, r_{i}}^{2}=\delta_{s, r}^{2}$ and $\delta_{r_{i}, d}^{2}=\delta_{r, d}^{2}$ for $i=1,2, \ldots, N$. Let $t_{1}=\sqrt{q_{1} q_{2} \delta_{s, r}^{2} \delta_{r, d}^{2}}$ and $t_{2}=\frac{1}{q_{2} \delta_{s, r}^{2}}+$ $\frac{1}{q_{1} \delta_{r, d}^{2}}$. The CDF and PDF of $\beta_{\max }$ can be written as shown in the two statements of (16), respectively, where $p_{\beta_{m}}($.$) is the$ PDF of $\beta_{m}$ (6) and it is given by (17). The probability of the direct-transmission mode can be obtained as shown in (18), where we approximated $K_{1}($.$) as in (15) and \beta_{s, d}$ is an exponential random variable with parameter $1 / \delta_{s, d}^{2}$. The probability of the relay-cooperation mode is $\operatorname{Pr}\left(\phi^{c}\right)=1-\operatorname{Pr}(\phi)$. Since the bandwidth efficiency of the direct-transmission mode is 1 SPCU, and that of the relay-cooperation mode is $1 / 2$ SPCU, thus the average bandwidth efficiency can be written as

$$
R=\operatorname{Pr}(\phi)+\frac{1}{2} \operatorname{Pr}\left(\phi^{c}\right),
$$

which results in the following theorem.

Theorem 1: The bandwidth efficiency of the multi-node relay-selection decode-and-forward symmetric cooperative scenario, employing $N$ relays, is approximated as shown in (20).

Fig. 3 depicts the bandwidth efficiency of the relay-selection assuming $\alpha=1$ and $r=0.5$ and the conventional cooperative schemes for different number of relays and unity channel variances. It is clear that the bandwidth efficiency decreases down to 0.5 as $N$ increases, because the probability of the direct-transmission mode decreases down to 0 as $N$ goes to $\infty$. Intuitively, increasing the number of relays increases the probability of having the optimal relay's metric higher than the source-destination channel gain. Furthermore, we plot the simulated bandwidth efficiency results for the proposed relay selection algorithm. Also, we plot the bandwidth efficiency of the conventional cooperative scheme, $R_{\text {conv }}=\frac{1}{N+1}$ SPCU, to show the significant increase in the bandwidth efficiency of the proposed relay-selection cooperative scenario over the

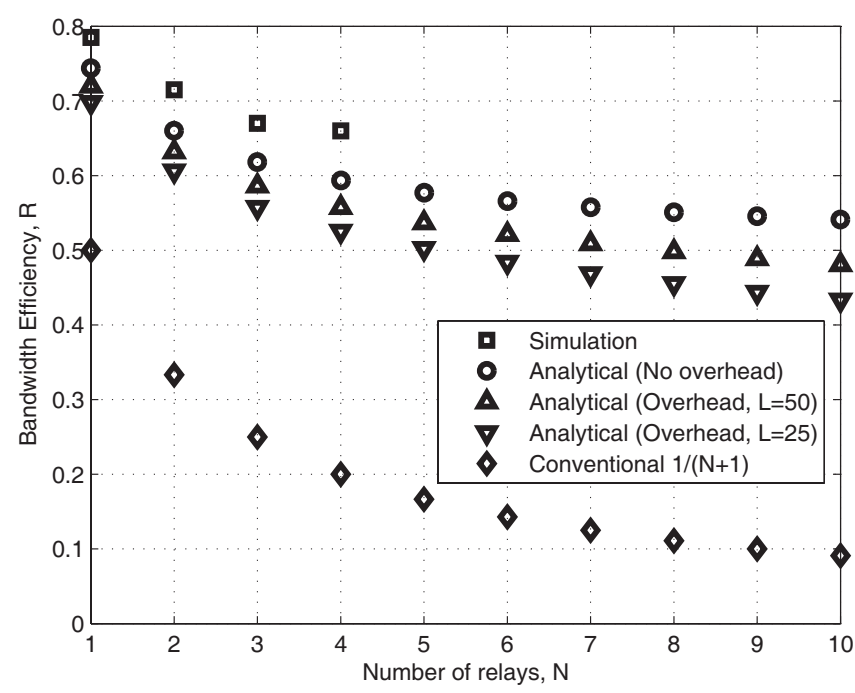

Fig. 3. Bandwidth efficiency dependence on the number of relays with QPSK modulation and unity channel variances, $\alpha=1$, and $r=0.5$.

conventional cooperative scheme.

In the sequel, we address the system overhead issue. Particularly, we compute the bandwidth efficiency of the proposed scheme taking into consideration the overhead. We assume slow fading channels, where the channels are constant during a transmission block (channel coherence time) and may vary from a block to another. For moderate to high data rate the block length $L$, measured in terms of the number of transmitted data symbols during the channel coherence time, can be relatively large. We assume that the feedback channel is an orthogonal channel. Each relay sends a quantized version of the harmonic mean function to the source. For simplicity, we assume that it uses the same modulation order (M-PSK) as that of the original data. For slowly varying channels, we expect that the change in each relay's metric will be relatively small across each two consecutive blocks. In this case, it is more reasonable to modulate the difference in the relays' metrics 


$$
R \approx \frac{1}{2} \cdot\left(1+\sum_{n=0}^{N}\left(\begin{array}{l}
N \\
n
\end{array}\right)(-1)^{n} \frac{2 \alpha}{2 \alpha+\left(\frac{1-r}{B \delta_{s, r}^{2}}+\frac{r}{A^{2} \delta_{r, d}^{2}}\right) r \delta_{s, d}^{2} n}\right) \quad S P C U
$$

rather than the absolute values. This helps in having a small quantization error and the performance can be very close to the one without quantization. Thus, the overhead for each block is $N+1$ symbols, where $N$ symbols are transmitted from the $N$ relays and the last symbol is sent from the source to indicate whether to cooperate or not and which relay to cooperate with in case of cooperation.

Taking the overhead into consideration the bandwidth efficiency, given previously in (19), can be recalculated as

$$
\begin{aligned}
& R_{\text {overhead }} \\
& =\frac{L}{L+(N+1)} \operatorname{Pr}(\phi)+\frac{L}{2 L+(N+1)} \operatorname{Pr}\left(\phi^{c}\right),
\end{aligned}
$$

where $\operatorname{Pr}(\phi)$ is the direct transmission probability. Fig. 3 depicts the effect of the overhead on the bandwidth efficiency for different block lengths. For moderate block length, $L=25$, and $N=3$ relays the bandwidth efficiency is 0.56 , while it is $1 / 3$ for the conventional scheme. Hence, an increase of $68 \%$ is achieved by our proposed algorithm.

An alternative protocol to send the feedback information can be explained as follows. As we mentioned, we assume slow fading channel that can be statistically modeled. For instance, we assume that the channel follows Jakes Rayleigh fading model. The Jakes tap gain process is stationary and can be modeled as an auto-regressive (AR) model. Thus, each relay can send the AR coefficients representing its relaydestination channels to the source. The source utilizes these parameters to predict the relay-destination channel for each relay. Obviously, these AR coefficients are sent at the start of the transmission. This reduces the overhead significantly compared to the scheme explained above. In order to reduce the prediction error, each relay updates the source with its current instantaneous relay-destination channel with a period that lasts for a certain number of transmission blocks that depends on how slow the channel varies. The source can estimate its source-relay channel with each relay with no extra cost as follows. In the conventional scheme, it is assumed that each relay broadcasts a pilot signal so that the destination can estimate the relay-destination channel. The destination utilizes the estimated relay-destination channel in order to decode the signal received from each relay. So, the source can make use of these pilots too to estimate its source-relay channels. Then, the source computes all the relays metrics based on the estimated source-relay channels and the predicted relay-destination channels. It determines the optimal relay and decides whether to cooperate with it or not based on its sourcedestination channel gain.

\section{B. SER Analysis and Upper Bound}

In order to prove full diversity order for our proposed scheme, we make use of the following lemma.
Lemma 1: For any $x, y$, and $N$

$$
\sum_{n=0}^{N}\left(\begin{array}{l}
N \\
n
\end{array}\right)(-1)^{n} \frac{1}{x+n y}=\frac{(N) ! y^{N}}{\prod_{n=0}^{N}(x+n y)}
$$

Proof of Lemma 1 is given in the Appendix.

The probability of symbol error, or SER, is defined as

$$
\operatorname{Pr}(e)=\operatorname{Pr}(e / \phi) \cdot \operatorname{Pr}(\phi)+\operatorname{Pr}\left(e / \phi^{c}\right) \cdot \operatorname{Pr}\left(\phi^{c}\right),
$$

where $\operatorname{Pr}(e / \phi) \cdot \operatorname{Pr}(\phi)$ represents the SER of the directtransmission mode and $\operatorname{Pr}\left(e / \phi^{c}\right) \cdot \operatorname{Pr}\left(\phi^{c}\right)$ represents the relaycooperation mode SER. The SER of the direct-transmission mode can be calculated as follows. First, the instantaneous direct-transmission SNR is $\gamma^{\phi}=\frac{P \beta_{s, d}}{N_{0}}$. The conditional direct-transmission SER can be written, as given in [17], as

$\operatorname{Pr}\left(e / \phi, \beta_{s, d}\right)=\Psi\left(\gamma^{\phi}\right)=\frac{1}{\pi} \int_{0}^{\frac{(M-1) \pi}{M}} \exp \left(-\frac{b \gamma^{\phi}}{\sin ^{2} \theta}\right) d \theta$

where $b=\sin ^{2}(\pi / M)$. Thus, the SER of the directtransmission is calculated as

$$
\begin{aligned}
& \operatorname{Pr}(e / \phi) \operatorname{Pr}(\phi) \\
& =\int_{0}^{\infty} \operatorname{Pr}\left(e / \phi, \beta_{s, d}\right) \operatorname{Pr}\left(\phi / \beta_{s, d}\right) \cdot p_{\beta_{s, d}}\left(\beta_{s, d}\right) d \beta_{s, d} \\
& \approx \sum_{n=0}^{N}\left(\begin{array}{l}
N \\
n
\end{array}\right)(-1)^{n} F_{1}\left(1+\frac{t_{2} \delta_{s, d}^{2} n}{2 \alpha}+\frac{b P}{N_{0} \sin ^{2} \theta} \delta_{s, d}^{2}\right),
\end{aligned}
$$

where we applied the approximation in (15) and $F_{1}(x(\theta))=$ $\frac{1}{\pi} \int_{0}^{\frac{(M-1) \pi}{M}} \frac{1}{x(\theta)} d \theta$.

For the relay-cooperation mode, maximal-ratio combining (MRC) [13] is applied at the destination. The output of the MRC [13] can be written as

$$
y^{\phi^{c}}=\frac{\sqrt{P_{1}} h_{s, d}^{*}}{N_{0}} y_{s, d}^{\phi^{c}}+\frac{\sqrt{\tilde{P}_{2}} h_{r, d}^{*}}{N_{0}} y_{r, d}^{\phi^{c}},
$$

and the instantaneous SNR of the MRC output is given by $\gamma^{\phi^{c}}=\frac{P_{1} \beta_{s, d}+\tilde{P}_{2} \beta_{r, d}}{N_{0}}$. Taking into account the two scenarios of $\tilde{P}_{2}=0$ and $\tilde{P}_{2}=P_{2}$, the conditional SER of the relaycooperation mode is given, as in [4], as shown in (27).

Let $\operatorname{Pr}\left(A / \phi^{c}, \beta_{s, d}, \beta_{s, r}, \beta_{r, d}\right)=\Psi\left(\gamma^{\phi^{c}}\right) \Psi\left(\frac{\dot{P}_{1} \beta_{s, r}}{N_{0}}\right)$ and $\operatorname{Pr}\left(B / \phi^{c}, \beta\right)=\Psi\left(\gamma^{\phi^{c}}\right)$. Since the value of $\operatorname{Pr}\left(A / \phi^{c}, \beta_{s, d}, \overline{\beta_{s}, r}, \beta_{r, d}\right)$ can be expressed as shown in (28), thus,

$$
\begin{aligned}
& \operatorname{Pr}\left(A / \phi^{c}\right) \operatorname{Pr}\left(\phi^{c}\right) \\
& =\int_{\underline{\beta}} \operatorname{Pr}\left(A / \phi^{c}, \underline{\beta}\right) \operatorname{Pr}\left(\phi^{c} / \underline{\beta}\right) p_{\underline{\beta}}(\underline{\beta}) d \underline{\beta},
\end{aligned}
$$

where $\underline{\beta} \triangleq\left[\beta_{s, d}, \beta_{s, r}, \beta_{r, d}\right]$. Furthermore,

$$
\begin{aligned}
\operatorname{Pr}\left(\phi^{c} / \underline{\beta}\right) & =\operatorname{Pr}\left(\beta_{s, d}<\alpha \beta_{\max } / \beta_{s, d}, \beta_{s, r}, \beta_{r, d}\right) \\
& =U\left(\alpha \beta_{\max }-\beta_{s, d}\right),
\end{aligned}
$$




$$
\begin{aligned}
\operatorname{Pr}\left(e / \phi^{c}, \beta_{s, d}, \beta_{s, r}, \beta_{r, d}\right)= & \left.\Psi\left(\gamma^{\phi^{c}}\right)\right|_{\tilde{P}_{2}=0} \Psi\left(\frac{P_{1} \beta_{s, r}}{N_{0}}\right)+\left.\Psi\left(\gamma^{\phi^{c}}\right)\right|_{\tilde{P}_{2}=P_{2}}\left(1-\Psi\left(\frac{P_{1} \beta_{s, r}}{N_{0}}\right)\right) \\
\operatorname{Pr}\left(A / \phi^{c}, \beta_{s, d}, \beta_{s, r}, \beta_{r, d}\right)= & \frac{1}{\pi} \int_{\theta_{1}=0}^{\frac{(M-1) \pi}{M}} \exp \left(-\frac{b P_{1}}{N_{0} \sin ^{2} \theta_{1}} \beta_{s, d}\right) \exp \left(-\frac{b \tilde{P}_{2}}{N_{0} \sin ^{2} \theta_{1}} \beta_{r, d}\right) d \theta_{1} \\
& \times \frac{1}{\pi} \int_{\theta_{2}=0}^{\frac{(M-1) \pi}{M}} \exp \left(-\frac{b P_{1}}{N_{0} \sin ^{2} \theta_{2}} \beta_{s, r}\right) d \theta_{2}, \\
\operatorname{Pr}\left(A / \phi^{c}\right) \operatorname{Pr}\left(\phi^{c}\right)= & \int_{\underline{\beta}} \frac{1}{\pi^{2}} \int_{\theta_{1}=0}^{\frac{(M-1) \pi}{M}} \int_{\theta_{2}=0}^{\frac{(M-1) \pi}{M}} \exp \left(-P_{1} C\left(\theta_{1}\right) \beta_{s, d}\right) \exp \left(-\tilde{P}_{2} C\left(\theta_{1}\right) \beta_{r, d}\right) \\
\times & \exp \left(-P_{1} C\left(\theta_{2}\right) \beta_{s, r}\right) U\left(\alpha \beta_{\max }-\beta_{s, d}\right) p_{\underline{\beta}}(\underline{\beta}) d \theta_{2} d \theta_{1} d \underline{\beta}
\end{aligned}
$$

where $U($.$) is the unit step function. Substituting (28) and$ (30) into (29), we get (31), where $C(\theta)=\frac{b}{N_{0} \sin ^{2} \theta}$. Since $\beta_{s, d}, \beta_{s, r}$, and $\beta_{r, d}$ are statistically independent, thus

$$
\begin{aligned}
p_{\underline{\beta}}(\underline{\beta}) & =p_{\beta_{s, d}}\left(\beta_{s, d}\right) p_{\beta_{s, r}}\left(\beta_{s, r}\right) p_{\beta_{r, d}}\left(\beta_{r, d}\right) \\
& =p_{\beta_{s, d}}\left(\beta_{s, d}\right) p_{\underline{\tilde{\beta}}}(\underline{\tilde{\beta}}),
\end{aligned}
$$

where $\tilde{\beta} \triangleq\left[\beta_{s, r}, \beta_{r, d}\right]$. Integrating (31) with respect to $\beta_{s, d}$, we get (33).

It is difficult to get an exact expression of (33) for $\beta_{\max }$ defined in (8). Thus, we obtain an upper bound via a worstcase scenario. We replace $\beta_{s, r}$ and $\beta_{r, d}$ in (33) by their worstcast values in terms of $\beta_{\max }$. Then, we average (33) over $\beta_{\max }$ only. Since $\beta_{\max }=\mu_{H}\left(q_{1} \beta_{r, d}, q_{2} \beta_{s, r}\right)$, we can write $\frac{1}{\beta_{\max }}=\frac{1}{2 q_{2} \beta_{s, r}}+\frac{1}{2 q_{1} \beta_{r, d}}$. Then, we replace $\beta_{s, r}$ and $\beta_{r, d}$ by their worst values in terms of $\beta_{\max }$ as $\beta_{s, r} \longrightarrow \frac{\beta_{\max }}{2 q_{2}}$ and $\beta_{r, d} \longrightarrow \frac{\beta_{\max }}{2 q_{1}}$. Thus, (33) can be upper bounded as shown in (34), where $M_{\beta_{\max }}($.$) is the moment generation function$ (MGF) of $\beta_{\max }$ and it can be approximated as

$$
M_{\beta_{\max }}(\gamma) \approx N \sum_{n=0}^{N-1}\left(\begin{array}{l}
N-1 \\
n
\end{array}\right)(-1)^{n} M_{\beta_{m}}\left(\gamma+\frac{n t_{2}}{2}\right),
$$

where we have applied (15) and $M_{\beta_{m}}$ (.) is the MGF of $\beta_{m}$. It was shown in [14] that for two independent exponential random variables with parameters $\lambda_{1}$ and $\lambda_{2}$, the MGF of their harmonic mean function is written as shown in (36), where $E_{\beta_{m}}($.$) represents the statistical average with respect$ to $\beta_{m}$ and ${ }_{2} F_{1}(., . ; . ;$.$) is the Gauss' hypergeometric function$ defined in [[16], (15.1.1)]. Following similar steps as done in (28)-(34), we get (37).

The unconditional SER of the relay-cooperation mode can be written from (27) as

$$
\begin{aligned}
& \operatorname{Pr}\left(e / \phi^{c}\right) \operatorname{Pr}\left(\phi^{c}\right) \\
& =\left.\operatorname{Pr}\left(A / \phi^{c}\right) \operatorname{Pr}\left(\phi^{c}\right)\right|_{\tilde{P}_{2}=0}-\left.\operatorname{Pr}\left(A / \phi^{c}\right) \operatorname{Pr}\left(\phi^{c}\right)\right|_{\tilde{P}_{2}=P_{2}} \\
& +\left.\operatorname{Pr}\left(B / \phi^{c}\right) \operatorname{Pr}\left(\phi^{c}\right)\right|_{\tilde{P}_{2}=P_{2}} .
\end{aligned}
$$

Since $\left.\operatorname{Pr}\left(A / \phi^{c}\right) \operatorname{Pr}\left(\phi^{c}\right)\right|_{\tilde{P}_{2}=P_{2}}$ in (33) is a positive value, therefore an upper bound on the SER of the relay-cooperation mode can be obtained by removing this term from (38).
Moreover, we can remove the subtracted terms in (34) and (37). Therefore, an upper bound on the total SER can be obtained by adding (25), (34), and (37), after removing the subtracted terms, as shown in (39), where we applied Lemma 1 for the direct-transmission SER in (25).

In order to show that full diversity order is achieved, we derive an upper bound on the SER performance at high SNR. At high SNR, we neglect the terms 1 and $\left(1+\frac{t_{2} \delta_{s, d}^{2} n}{2 \alpha}\right)$ in (39). Thus, the SER upper bound is written as shown in (40).

The SER upper bound expression in (40) is in terms of the MGF $M_{\beta_{m}}($.$) , which is mathematically intractable. In [5],$ the authors have presented an approximation to the MGF of two independent exponential random variables at high enough SNR as

$$
M_{\beta_{m}}(\gamma) \approx \frac{q_{1} \delta_{r, d}^{2}+q_{2} \delta_{s, r}^{2}}{2 \gamma} .
$$

Using the MGF approximation given in (41) and Lemma 1, we obtain (42), where

$$
I(p)=\frac{1}{\pi} \int_{\theta=0}^{\frac{(M-1) \pi}{M}} \sin ^{p} \theta d \theta .
$$

Neglecting the term $\left(n t_{2}\right)$ at high SNR, we get (44). Replacing $q_{1}=\frac{A^{2}}{r^{2}}, q_{2}=\frac{B}{r(1-r)}$, and $t_{2}=\frac{1}{q_{2} \delta_{s, r}^{2}}+\frac{1}{q_{1} \delta_{r, d}^{2}}$, and using $P_{1}=r P$ and $P_{2}=(1-r) P$, we get the following theorem.

Theorem 2: At high SNR $\gamma=\frac{P}{N_{0}}$, the SER of the multinode relay-selection decode-and-forward symmetric cooperative scenario, utilizing $N$ relays, is upper bounded by

$$
\operatorname{Pr}(e) \leq(C G \cdot \gamma)^{-(N+1)}
$$

where the value of $C G$ is given in (46).

As defined earlier, the diversity order is $d=-\lim _{S N R \longrightarrow \infty} \log (\operatorname{Pr}(e)) / \log (S N R)$. By substituting (44), the diversity order of the proposed algorithm is $N+1$.

\section{Single-relay Scenario: When to Cooperate?}

In the conventional single-relay decode-and-forward cooperative scheme, one symbol is sent each two time slots. Hence, the bandwidth efficiency is 0.5 SPCU. In the singlerelay scenario, it is meaningful to consider only the question: 


$$
\begin{aligned}
& \operatorname{Pr}\left(A / \phi^{c}\right) \operatorname{Pr}\left(\phi^{c}\right)=\int_{\tilde{\tilde{\beta}}} \frac{1}{\pi^{2}} \int_{\theta_{1}=0}^{\frac{(M-1) \pi}{M}} \int_{\theta_{2}=0}^{\frac{(M-1) \pi}{M}} \frac{1-\exp \left(-\left(P_{1} C\left(\theta_{1}\right)+\frac{1}{\delta_{s, d}^{2}}\right) \alpha \beta_{\max }\right)}{1+P_{1} C\left(\theta_{1}\right) \delta_{s, d}^{2}} p_{\tilde{\tilde{\beta}}}(\underline{\tilde{\beta}}) \\
& \times \exp \left(-\left(\tilde{P}_{2} C\left(\theta_{1}\right) \beta_{r, d}+P_{1} C\left(\theta_{2}\right) \beta_{s, r}\right)\right) d \theta_{2} d \theta_{1} d \underline{\tilde{\beta}} \\
& \operatorname{Pr}\left(A / \phi^{c}\right) \operatorname{Pr}\left(\phi^{c}\right) \leq \frac{1}{\pi^{2}} \int_{\theta_{1}=0}^{\frac{(M-1) \pi}{M}} \frac{d \theta_{1}}{1+P_{1} C\left(\theta_{1}\right) \delta_{s, d}^{2}} \int_{\theta_{2}=0}^{\frac{(M-1) \pi}{M}}\left(M_{\beta_{\max }}\left(\frac{\tilde{P}_{2} C\left(\theta_{1}\right)}{2 q_{1}}+\frac{P_{1} C\left(\theta_{2}\right)}{2 q_{2}}\right)\right. \\
& \left.-M_{\beta_{\max }}\left(\left(P_{1} C\left(\theta_{1}\right)+\frac{1}{\delta_{s, d}^{2}}\right) \alpha+\frac{\tilde{P}_{2} C\left(\theta_{1}\right)}{2 q_{1}}+\frac{P_{1} C\left(\theta_{2}\right)}{2 q_{2}}\right)\right) d \theta_{2} \\
& M_{\beta_{m}}(\gamma)=E_{\beta_{m}}\left(\exp \left(-\gamma \beta_{m}\right)\right)=\frac{16 \lambda_{1} \lambda_{2}}{3\left(\lambda_{1}+\lambda_{2}+2 \sqrt{\lambda_{1} \lambda_{2}}+\gamma\right)^{2}} \\
& \times\left(\frac{4\left(\lambda_{1}+\lambda_{2}\right){ }_{2} F_{1}\left(3, \frac{3}{2} ; \frac{5}{2} ; \frac{\lambda_{1}+\lambda_{2}-2 \sqrt{\lambda_{1} \lambda_{2}}+\gamma}{\lambda_{1}+\lambda_{2}+2 \sqrt{\lambda_{1} \lambda_{2}}+\gamma}\right)}{\left(\lambda_{1}+\lambda_{2}+2 \sqrt{\lambda_{1} \lambda_{2}}+\gamma\right)}+{ }_{2} F_{1}\left(2, \frac{1}{2} ; \frac{5}{2} ; \frac{\lambda_{1}+\lambda_{2}-2 \sqrt{\lambda_{1} \lambda_{2}}+\gamma}{\lambda_{1}+\lambda_{2}+2 \sqrt{\lambda_{1} \lambda_{2}}+\gamma}\right)\right) \\
& \operatorname{Pr}\left(B / \phi^{c}\right) \operatorname{Pr}\left(\phi^{c}\right) \leq \frac{1}{\pi} \int_{\theta=0}^{\frac{(M-1) \pi}{M}}\left(\frac{M_{\beta_{\max }}\left(\frac{\tilde{P}_{2} C(\theta)}{2 q_{1}}\right)}{1+P_{1} C(\theta) \delta_{s, d}^{2}}-\frac{M_{\beta_{\max }}\left(\left(P_{1} C(\theta)+\frac{1}{\delta_{s, d}^{2}}\right) \alpha+\frac{\tilde{P}_{2} C(\theta)}{2 q_{1}}\right)}{1+P_{1} C(\theta) \delta_{s, d}^{2}}\right) d \theta \\
& \operatorname{Pr}(e) \leq N !\left(\frac{t_{2} \delta_{s, d}^{2}}{2 \alpha}\right)^{N} F_{1}\left(\prod_{n=0}^{N}\left(1+\frac{t_{2} \delta_{s, d}^{2} n}{2 \alpha}+\frac{b P \delta_{s, d}^{2}}{N_{0} \sin ^{2} \theta}\right)\right)+\sum_{n=0}^{N-1}\left(\begin{array}{l}
N-1 \\
n
\end{array}\right) \frac{(-1)^{n}}{\pi} \\
& \times \int_{\theta_{1}=0}^{\frac{(M-1) \pi}{M}}\left(\frac{M_{\beta_{m}}\left(\frac{b P_{2}}{2 q_{1} N_{0} \sin ^{2} \theta_{1}}+\frac{n t_{2}}{2}\right)}{1+\frac{b P_{1} \delta_{s, d}^{2}}{N_{0} \sin ^{2} \theta_{1}}}+\frac{\frac{1}{\pi} \int_{\theta_{2}=0}^{\frac{(M-1) \pi}{M}} M_{\beta_{m}}\left(\frac{b P_{1}}{2 q_{2} N_{0} \sin ^{2} \theta_{2}}+\frac{n t_{2}}{2}\right) d \theta_{2}}{1+\frac{b P_{1} \delta_{s, d}^{2}}{N_{0} \sin ^{2} \theta_{1}}}\right) d \theta_{1} \\
& \operatorname{Pr}(e) \leq N !\left(\frac{t_{2} \delta_{s, d}^{2}}{2 \alpha}\right)^{N} F_{1}\left(\left(\frac{b P}{N_{0} \sin ^{2} \theta} \delta_{s, d}^{2}\right)^{N+1}\right)+\sum_{n=0}^{N-1}\left(\begin{array}{l}
N-1 \\
n
\end{array}\right)(-1)^{n} \frac{1}{\pi} \int_{\theta_{1}=0}^{\frac{(M-1) \pi}{M}} \frac{N_{0} \sin ^{2} \theta_{1}}{b P_{1} \delta_{s, d}^{2}} \\
& \times\left(M_{\beta_{m}}\left(\frac{b P_{2}}{2 q_{1} N_{0} \sin ^{2} \theta_{1}}+\frac{n t_{2}}{2}\right)+\frac{1}{\pi} \int_{\theta_{2}=0}^{\frac{(M-1) \pi}{M}} M_{\beta_{m}}\left(\frac{b P_{1}}{2 q_{2} N_{0} \sin ^{2} \theta_{2}}+\frac{n t_{2}}{2}\right) d \theta_{2}\right) d \theta_{1} \\
& \operatorname{Pr}(e) \leq N !\left(\frac{N_{0}}{b P}\right)^{N+1}\left(\frac{t_{2}}{2 \alpha}\right)^{N} \frac{I(2 N+2)}{\delta_{s, d}^{2}}+N ! t_{2}^{N-1}\left(q_{1} \delta_{r, d}^{2}+q_{2} \delta_{s, r}^{2}\right) \frac{1}{\pi} \int_{\theta_{1}=0}^{\frac{(M-1) \pi}{M}} \frac{N_{0} \sin ^{2} \theta_{1}}{b P_{1} \delta_{s, d}^{2}} \\
& \times\left(\frac{1}{\prod_{n=0}^{N-1}\left(\frac{b P_{2}}{q_{1} N_{0} \sin ^{2} \theta_{1}}+n t_{2}\right)}+\frac{1}{\pi} \int_{\theta_{2}=0}^{\frac{(M-1) \pi}{M}} \frac{1}{\prod_{n=0}^{N-1}\left(\frac{b P_{1}}{q_{2} N_{0} \sin ^{2} \theta_{2}}+n t_{2}\right)} d \theta_{2}\right) d \theta_{1} \\
& \operatorname{Pr}(e) \leq N !\left(\frac{N_{0}}{b P}\right)^{N+1}\left(\frac{t_{2}}{2 \alpha}\right)^{N} \frac{I(2 N+2)}{\delta_{s, d}^{2}}+N !\left(\frac{N_{0}}{b}\right)^{N+1} t_{2}^{N-1} \frac{\left(q_{1} \delta_{r, d}^{2}+q_{2} \delta_{s, r}^{2}\right)}{P_{1} \delta_{s, d}^{2}} \\
& \times\left(\left(\frac{q_{1}}{P_{2}}\right)^{N} I(2 N+2)+\left(\frac{q_{2}}{P_{1}}\right)^{N} A I(2 N)\right)
\end{aligned}
$$




$$
\begin{gathered}
C G=\left(\frac{N !\left(\frac{r(1-r)}{B \delta_{s, r}^{2}}+\frac{r^{2}}{A^{2} \delta_{r, d}^{2}}\right)^{N-1}}{b^{N+1} \delta_{s, d}^{2}}\right)^{-\frac{1}{(N+1)}} \\
\times\left(\frac{\left(\frac{r(1-r)}{B \delta_{s, r}^{2}}+\frac{r^{2}}{A^{2} \delta_{r, d}^{2}}\right) I(2 N+2)}{(2 \alpha)^{N}}+\frac{\left(\frac{A^{2} \delta_{r, d}^{2}}{r^{2}}+\frac{B \delta_{s, r}^{2}}{r(1-r)}\right)\left(A^{2 N} I(2 N+2)+B^{N} A I(2 N)\right)}{r^{N+1}(1-r)^{N}}\right)^{-\frac{1}{(N+1)}} .
\end{gathered}
$$

"When to cooperate?", as only one relay is available. Based on the general multi-node scheme described in Section II-C, the proposed single-relay scheme is described as follows.

First, the relay calculates the scaled harmonic mean function of its source-relay and relay-destination instantaneous channel gains (7), then sends it to the source. The source decides when to cooperate by taking the ratio between the sourcedestination channel gain and the relay's metric and comparing it to the cooperation threshold. If this ratio is greater than or equal to the cooperation threshold, then the source sends its information to the destination directly without the need for the relay. Otherwise, the source employs the relay in forwarding its information to the destination as in the conventional cooperative scheme. The source broadcasts its decision before the start of the data transmission.

In the sequel, we calculate the bandwidth efficiency and the SER of the proposed single-relay scheme. By substituting $N=1$ in Theorem 1, the bandwidth efficiency of the relayselection decode-and-forward cooperative scenario, utilizing single relay, can be approximated as

$$
R \approx \frac{\alpha+\left(\frac{1-r}{B \delta_{s, r}^{2}}+\frac{r}{A^{2} \delta_{r, d}^{2}}\right) r \delta_{s, d}^{2}}{2 \alpha+\left(\frac{1-r}{B \delta_{s, r}^{2}}+\frac{r}{A^{2} \delta_{r, d}^{2}}\right) r \delta_{s, d}^{2}} \quad S P C U .
$$

It is clear that the bandwidth efficiency is $R \geq 0.5$. By substituting $N=1$ in Theorem 2, the SER of the singlerelay relay-selection decode-and-forward cooperative scheme is upper bounded as $\operatorname{Pr}(e) \leq(C G \cdot \gamma)^{-2}$, where CG denotes the coding gain and is equal to

$$
C G=\sqrt{\frac{b^{2} \delta_{s, d}^{2}}{B\left(\frac{\frac{r(1-r)}{B \delta_{s, r}^{2}}+\frac{r^{2}}{A^{2} \delta_{r, d}^{2}}}{2 \alpha}+\frac{2 A^{2}\left(\frac{A^{2} \delta_{r, d}^{2}}{r^{2}}+\frac{B \delta_{s, r}^{2}}{r(1-r)}\right)}{r^{2}(1-r)}\right)}} .
$$

Note that $I(2)=\frac{1}{\pi} \int_{0}^{\frac{(M-1) \pi}{M}} \sin ^{2} \theta \quad d \theta=A$ and $\frac{1}{\pi} \int_{0}^{\frac{(M-1) \pi}{M}} \sin ^{4} \theta d \theta=B$ as defined in (2).

\section{Power Allocation and Cooperation Threshold}

In this section, an analytical expression of the optimum power allocation is derived, and bandwidth efficiency-SER tradeoff curves are shown to obtain the cooperation threshold. We clarify that as the cooperation threshold $\alpha$ increases, the probability of choosing the relay-cooperation mode increases. Therefore, the bandwidth efficiency and the SER, given by (20) and (44), respectively, decrease monotonically with $\alpha$. In addition, the bandwidth efficiency is a monotonically increasing or decreasing function of the power ratio $r=P_{1} / P$, depending on the channel variances. On the contrary, there exists an optimum power ratio $r^{*}$, which minimizes the SER. We determine the optimum power allocation as follows.

In the direct-transmission mode, all the power is transmitted through the source-destination channel. In the relaycooperation mode, we determine the optimum powers $P_{1}$ and $P_{2}$ which minimize the SER upper bound expression in (44) subject to constraint $P_{1}+P_{2}=P$. Substituting (5) into (44), we can approximate ${ }^{2}$ the optimization problem as shown in (49). By substituting $r=P_{1} / P$ into (49), we get (50). Solving (50) using the standard lagrangian method results in the following theorem.

Theorem 3: The optimum power allocation of the multinode relay-selection decode-and-forward symmetric cooperative scenario, employing $N$ relays, is obtained as

$$
\begin{aligned}
& P_{1}=\frac{1-\frac{N X_{1}}{2(N+1) X_{2}}+\sqrt{1+\frac{(N+2) X_{1}}{(N+1) X_{2}}+\left(\frac{N X_{1}}{2(N+1) X_{2}}\right)^{2}}}{2-\frac{N X_{1}}{2(N+1) X_{2}}+\sqrt{1+\frac{(N+2) X_{1}}{(N+1) X_{2}}+\left(\frac{N X_{1}}{2(N+1) X_{2}}\right)^{2}}} P, \\
& P_{2}=\frac{1}{2-\frac{N X_{1}}{2(N+1) X_{2}}+\sqrt{1+\frac{(N+2) X_{1}}{(N+1) X_{2}}+\left(\frac{N X_{1}}{2(N+1) X_{2}}\right)^{2}}} P,
\end{aligned}
$$

where

$$
\begin{aligned}
& X_{1}=\left(A^{2 N+2} I(2 N+2)+A^{3} B^{N} I(2 N)\right) \delta_{r, d}^{2}, \\
& X_{2}=\left(A^{2 N} B I(2 N+2)+A B^{N+1} I(2 N)\right) \delta_{s, r}^{2} .
\end{aligned}
$$

Theorem 3 shows that the optimum power allocation does not depend on the source-destination channel variance. It depends basically on the modulation order $M$ and the sourcerelay and relay-destination channel variances. If $\delta_{r, d}^{2} \gg \delta_{s, r}^{2}$ then $P_{1}$ goes to $P$ and $P_{2}$ goes to zero. Intuitively, this is because the source-relay link is of bad quality. Thus, it is reasonable to send the total power through the sourcedestination channel. In addition, if $\delta_{s, r}^{2} \gg \delta_{r, d}^{2}$ then $P_{1}$ goes to $P / 2$ and $P_{2}$ goes to $P / 2$ as well, which is expected because if the source-relay channel is so good, then the symbols will be received correctly by the relay with high probability. Thus, the relay will be almost the same as the source, thus both source and relay share the power equally.

The obtained optimum power ratio will be used to get the cooperation threshold as follows. Fig. 4 depicts the bandwidth efficiency-SER tradeoff curves for different number of relays at SNR equal to $20 \mathrm{~dB}$ and $25 \mathrm{~dB}$. This tradeoff is the achievable bandwidth efficiency and SER for different values of cooperation threshold. At a certain SER value, the

\footnotetext{
${ }^{2}$ It can be shown that this approximation has a very minor effect on the value of the optimum power ratio.
} 


$$
\begin{aligned}
& \min _{P_{1}}\left(\frac{A^{2} \delta_{r, d}^{2}}{r^{2} P_{1}}+\frac{B \delta_{s, r}^{2}}{r(1-r) P_{1}}\right) \cdot\left(\left(\frac{A^{2}}{r^{2} P_{2}}\right)^{N} I(2 N+2)+\left(\frac{B}{r(1-r) P_{1}}\right)^{N} A I(2 N)\right) \\
& \text { s.t. } P_{1}+P_{2}=P
\end{aligned}
$$

$$
\begin{array}{ll}
\min _{P_{1}} \frac{\left(A^{2 N+2} I(2 N+2)+A^{3} B^{N} I(2 N)\right) \delta_{r, d}^{2}}{P_{1}^{2 N+3} P_{2}^{N}}+\frac{\left(A^{2 N} B I(2 N+2)+A B^{N+1} I(2 N)\right) \delta_{s, r}^{2}}{P_{1}^{2 N+2} P_{2}^{N+1}} \\
\text { s.t. } P_{1}+P_{2}=P
\end{array}
$$

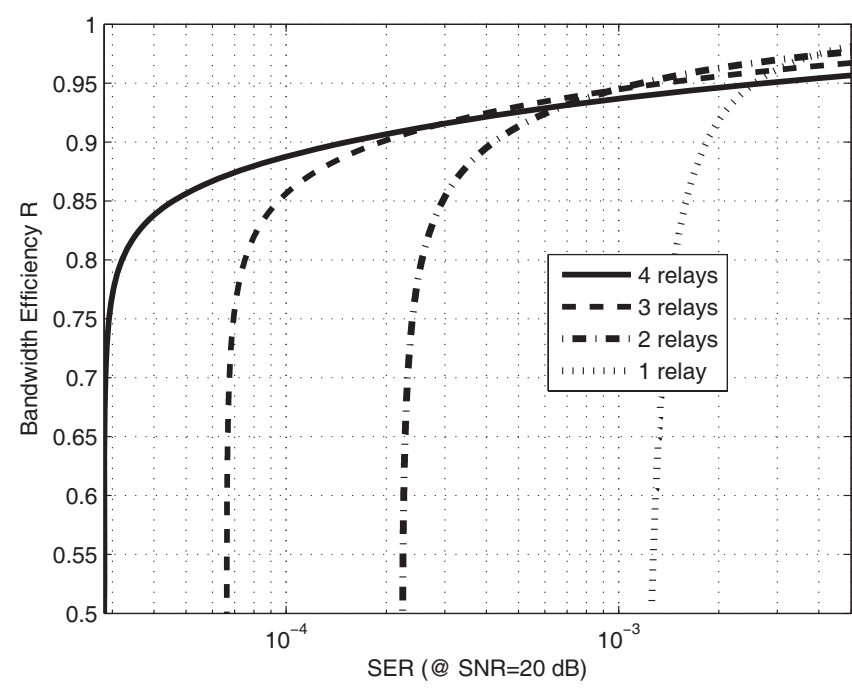

(a)

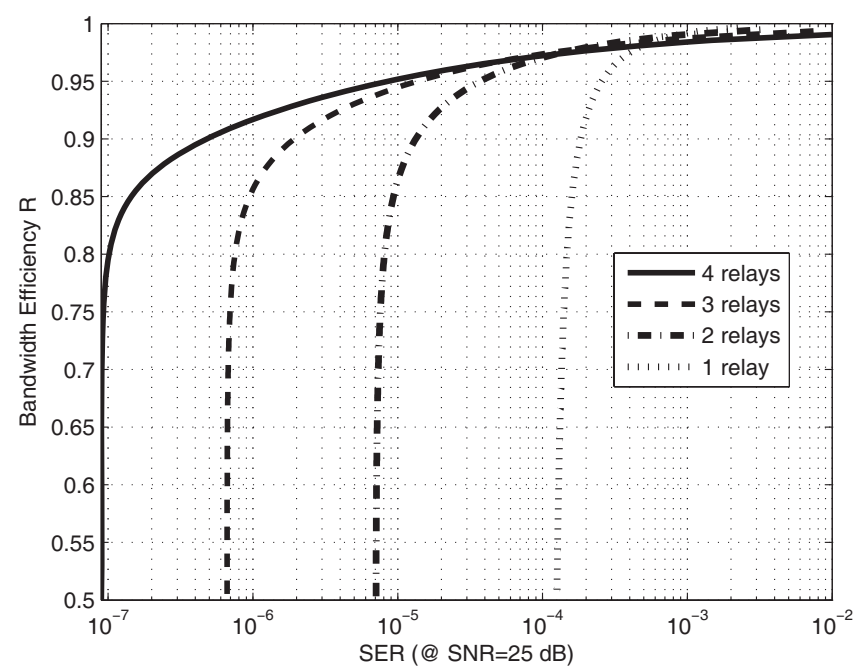

(b)

Fig. 4. Bandwidth efficiency versus SER at (a) $\mathrm{SNR}=20 \mathrm{~dB}$, (b) $\mathrm{SNR}=25$ dB.

maximum achievable bandwidth efficiency while guaranteeing full diversity order, can be obtained through Fig. 4. In Fig. 4, it is shown that the tradeoff achieved using four relays is the best among the plotted curves at low SER region. Moreover, it is clear in Fig. 4 (a) that the SER is almost constant at $2 \times 10^{-5}$ while the bandwidth efficiency increases from 0.5 to 0.8 SPCU for four relays. Thus, about $60 \%$ increase in the bandwidth efficiency can be achieved with the same SER performance.

In the sequel, we consider three different channel-variances cases for the single-relay case as follows. Case 1 which corresponds to the unity channel variances, where $\delta_{s, d}^{2}=$ $\delta_{s, r}^{2}=\delta_{r, d}^{2}=1$ and it is represented at the first row of Table I. Case 2 expresses a stronger relay-destination channel $\delta_{r, d}^{2}=10$, while case 3 expresses a stronger source-relay channel $\delta_{s, r}^{2}=10$. Cases 2 and 3 are represented at the second and third rows of Table I, respectively. Table I shows the optimum values of the power allocation ratio (51) for the three cases. Since we aim at maximizing both the coding gain and the bandwidth efficiency, we choose- as an example- an optimization metric, which is the product of the coding gain and bandwidth efficiency, to find the cooperation threshold. This optimization metric can be written as

$$
\max _{\alpha} C G \cdot R
$$

where R and CG are obtained from (20) and (46), respectively.

The cooperation threshold for the three different cases defined above are $\alpha_{o}=0.55, \alpha_{o}=0.09$, and $\alpha_{o}=0.14$, respectively. These values of cooperation thresholds result in bandwidth efficiencies equal to $R_{o}=0.8624, R_{o}=0.9075$, and $R_{o}=0.9443$ SPCU, respectively. Notably for $\delta_{r, d}^{2}=10$, the optimum power ratio is $r_{o}=0.7487$, which is greater than $r_{o}=0.6902$ for $\delta_{r, d}^{2}=1 ; \delta_{s, r}^{2}=1$ in both cases. This is in agreement with the conclusion that more power should be put for $P_{1}$ if $\delta_{r, d}^{2} \gg \delta_{s, r}^{2}$.

As shown in Fig. 5, increasing the number of relays affects the cooperation threshold values according to the $C G^{*} \mathrm{R}$ optimization criterion. Table II describes the effect of changing the number of relays on the power ratio, cooperation threshold, bandwidth efficiency, and the coding gain using the unity channel-variances case. A few comments on Table II are as follows. 1) The optimum power ratio is slightly decreasing with the number of relays. Because, increasing the number of relays will increase the probability of finding a better relay, which can receive the symbols from the source more correctly. Thus, it can send with almost equal power with the source. 2) The bandwidth efficiency is slightly decreasing with increasing the number of relays, because the probability that the sourcedestination channel is better than all the relays' metrics goes to 0 as $N$ goes to $\infty$.

\section{Simulation Results}

In this section, some computer simulations for the relayselection decode-and-forward cooperative system are presented to illustrate the previous theoretical analysis. It is 
TABLE I

Single-relay optimum values using the $(\mathrm{CG} * \mathrm{R})$ optimization criterion.

\begin{tabular}{|l|c|c|c|c|c|c|}
\hline$\delta_{s, d}^{2}$ & $\delta_{s, r}^{2}$ & $\delta_{r, d}^{2}$ & $r=P_{1} / P$ & $\alpha$ & Bandwidth Efficiency (R) & Coding Gain (CG) \\
\hline \hline 1 & 1 & 1 & 0.6902 & 0.55 & 0.8624 & 0.247 \\
\hline 1 & 1 & 10 & 0.7487 & 0.09 & 0.9075 & 0.1613 \\
\hline 1 & 10 & 1 & 0.6697 & 0.14 & 0.9443 & 0.0939 \\
\hline
\end{tabular}

TABLE II

$\mathrm{CG} * \mathrm{R}$ multi-node optimum values for unity channel variances.

\begin{tabular}{|c|c|c|c|c|}
\hline$N$ & Power ratio $(r)$ & Coop. threshold $(\alpha)$ & Bandwidth Efficiency (R) & Coding Gain (CG) \\
\hline \hline 1 & 0.6902 & 0.55 & 0.8624 & 0.247 \\
\hline 2 & 0.6826 & 0.41 & 0.8397 & 0.1512 \\
\hline 3 & 0.6787 & 0.35 & 0.8297 & 0.1046 \\
\hline 4 & 0.6764 & 0.32 & 0.82 & 0.0776 \\
\hline
\end{tabular}

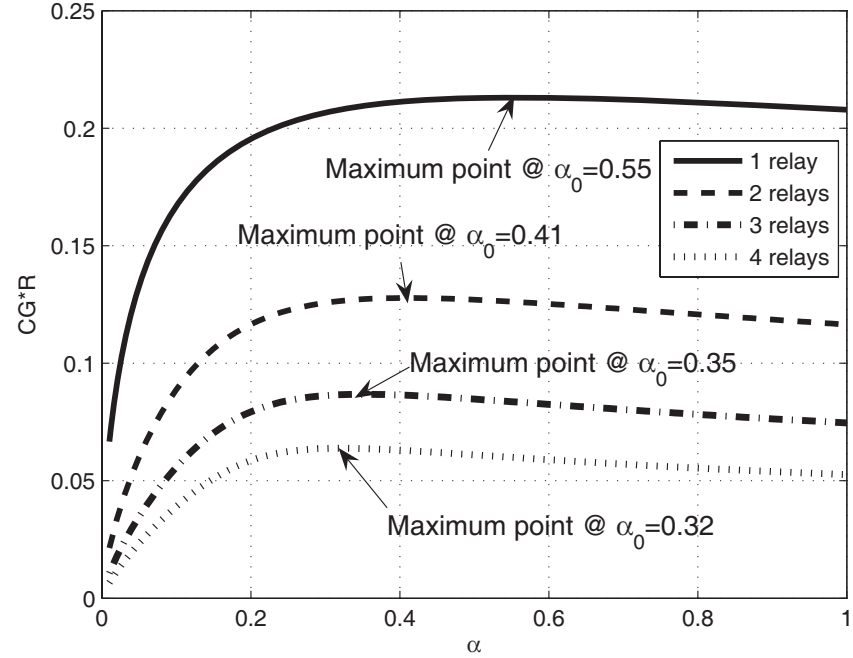

Fig. 5. Cooperation thresholds for different number of relays with unity channel variances.

assumed that the noise variance is set to $1, N_{0}=1$. For fair comparison, the SER curves are plotted as a function of $\mathrm{P} / N_{0}$. Finally, QPSK signalling is used in all the simulations.

Fig. 6 (a) depicts the simulated SER curves for the singlerelay relay-selection decode-and-forward cooperative scheme with unity channel variances. According to Table I, the optimum cooperation threshold and the optimum power ratio are $\alpha_{o}=0.55$ and $r_{o}=0.6902$, respectively. We plot the SER curve using the previous optimum values. Moreover, we plot the SER upper bound (44), which achieves full diversity order as was previously proven. Also, we plot the direct transmission curve which achieves diversity order 1, to show the advantage of using the cooperative scenario. Fig. 6 (b) shows the simulated SER curve for single-relay relayselection decode-and-forward cooperative scheme when the relay-destination channel is stronger, $\delta_{r, d}^{2}=10$. As shown in the second row of Table (I), the resultant power ratio, cooperation threshold, bandwidth efficiency, and coding gain are $0.7487,0.09,0.9075$, and 0.1613 , respectively. We plot the SER upper bound using the optimum power ratio. We have shown that the SER upper bound achieves full diversity order, which guarantees that the actual SER performance has full diversity order as well.

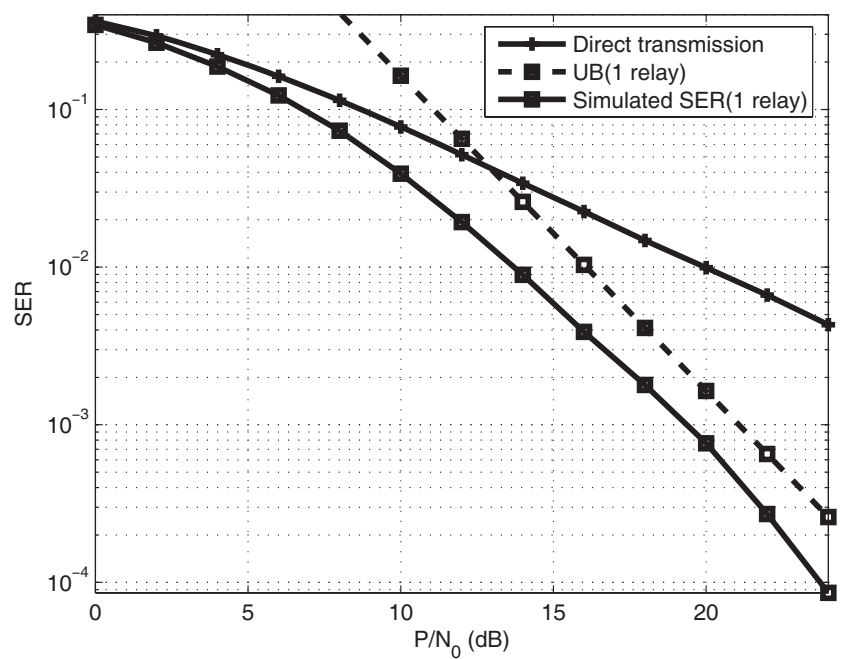

(a)

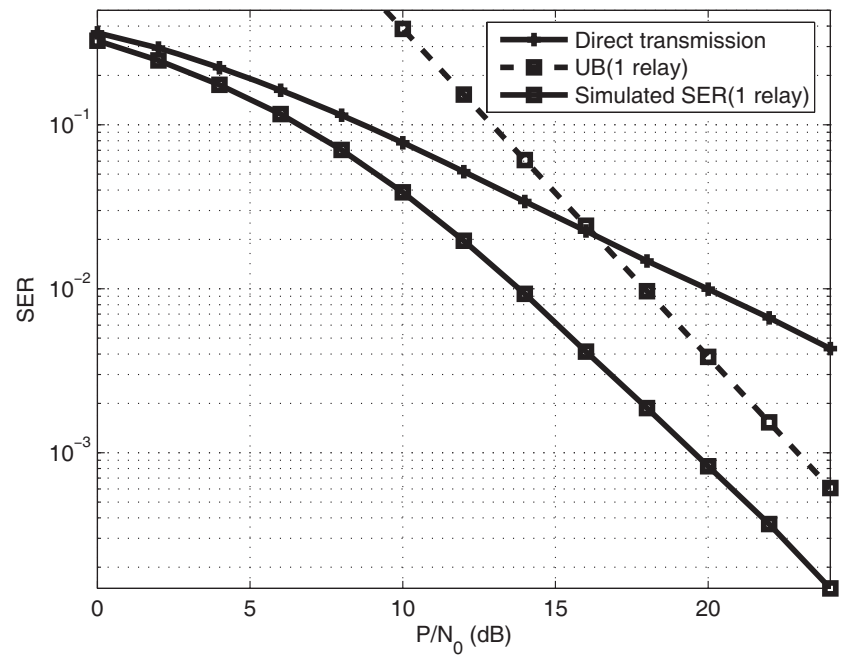

(b)

Fig. 6. SER simulated with optimum and equal power ratio, SER upper bound, and direct transmission curves for single-relay relay-selection decodeand-forward cooperative scheme with QPSK modulation, (a) $\alpha=0.55$, and unity channel variances, (b) $\alpha=0.09, \delta_{s, d}^{2}=1, \delta_{s, r}^{2}=1$, and $\delta_{r, d}^{2}=10$. 


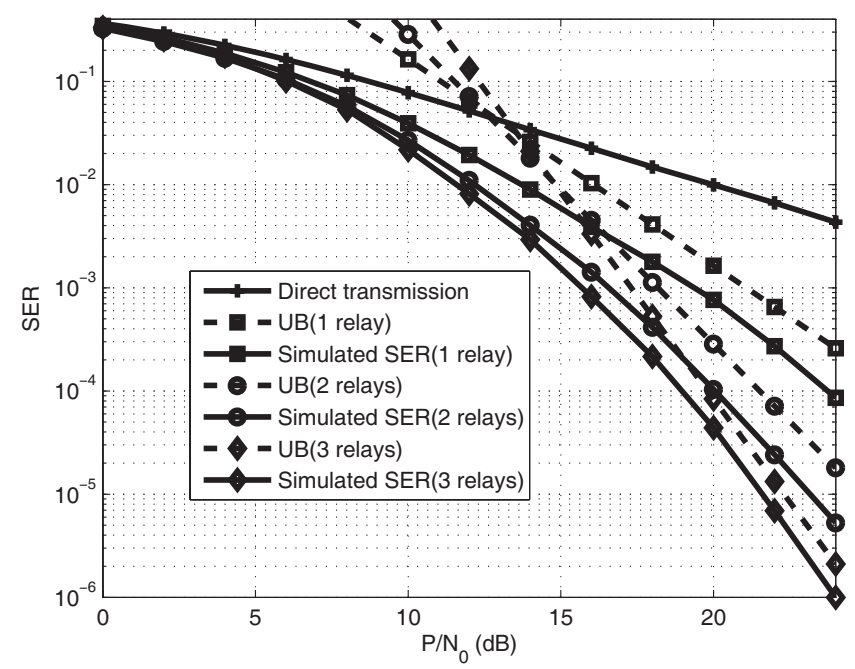

Fig. 7. SER simulated with optimum power ratio and SER upper bound curves for multi-node relay-selection decode-and-forward cooperative scheme with QPSK modulation and unity channel variances.

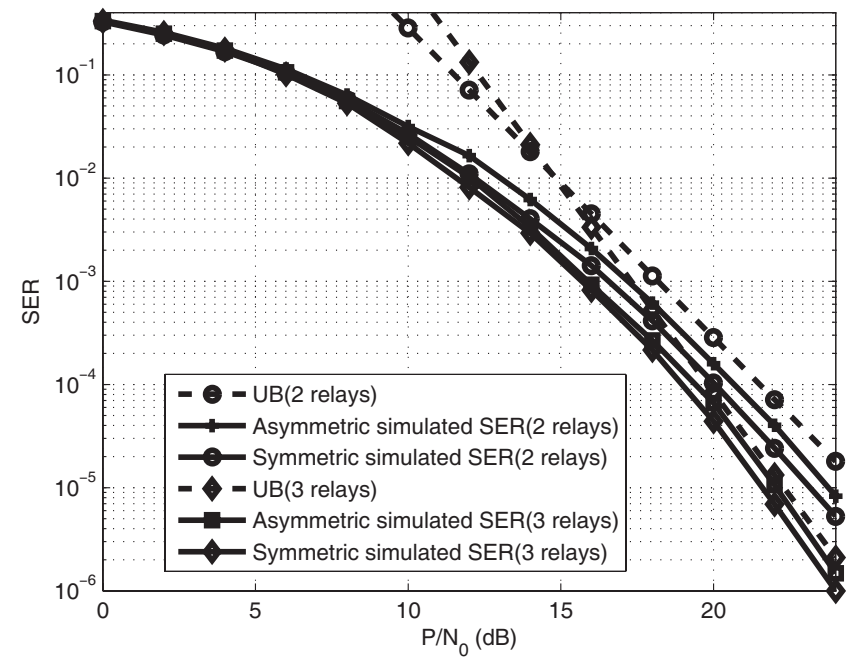

Fig. 8. SER simulated for symmetric (unity channel variance) and asymmetric cases for multi-node relay-selection decode-and-forward cooperative scheme with QPSK modulation.

Fig. 7 depicts the SER performance employing one, two, and three relays for unity channel variances. We plot the simulated SER curves using the optimum power ratios and the cooperation thresholds obtained in Table II. Moreover, we plot the SER upper bounds obtained in (44). It was shown in Theorem 2 that these upper bounds achieve full diversity order. It is obvious that the simulated SER curves are bounded by these upper bounds, hence they achieve full diversity order as well. The direct-transmission SER curve is plotted as well to show the effect of employing the relays in a cooperative way. Moreover, the simulated bandwidth efficiencies are $0.8973,0.8805$, and 0.8738 employing one, two, and three relays, respectively. These results are slightly higher than the analytical results shown in Table II.

Finally, we show some simulation results for the asymmetric case. In Fig. 8, we show the SER of the asymmetric case along with the symmetric results for two and three relays. For $N=2$ relays, the average channel gains are $\delta_{s, r_{1}}^{2}=\delta_{r_{2}, d}^{2}=1.5$ and $\delta_{s, r_{2}}^{2}=\delta_{r_{1}, d}^{2}=0.5$. For $N=3$, two of the three relays have the same average channel gains as in $N=2$. The third relay has $\delta_{s, r_{3}}^{2}=\delta_{r_{3}, d}^{2}=1$. We also compare the results with the symmetric case with unity channel variances. Hence, both the symmetric and asymmetric cases have the same average source-relay and relay-destination channel gains. The power ratio and cooperation threshold are obtained from Table II. As shown, the symmetric and asymmetric SER performance curves are very close to each other for both the two and three relays. More importantly, the asymmetric simulation results are upper bounded by the upper bound derived in Theorem 2 for the symmetric case.

\section{CONCLUSION}

In this paper, we have proposed a new multi-node relayselection decode-and-forward cooperative scenario, which utilizes the partial CSI available at the source and the relays. The main objective of this work is to achieve higher bandwidth efficiency and to guarantee full diversity order. We have defined the optimal relay as the one which has the maximum instantaneous scaled harmonic mean function of its sourcerelay and relay-destination channel gains among the $N$ helping relays. For the symmetric scenario, we have presented an approximate expression of the achievable bandwidth efficiency, which decreases with increasing the number of employed relays. Furthermore, we have derived the SER upper bound, which proves that full diversity order is guaranteed as long as there is cooperation. We have shown that the bandwidth efficiency is boosted up from 0.2 to $0.82 \mathrm{SPCU}$ for $N=4$ relays and unity channel variances case. As for the cooperation threshold, we have shown the bandwidth efficiency-SER tradeoff curve, which determines the cooperation threshold. Finally, we have presented some simulation results to verify the obtained analytical results.

\section{APPENDIX}

Proof of Lemma 1 is as follows. First, we prove that

$$
\sum_{n=0}^{N} \frac{\left(\begin{array}{l}
N \\
n
\end{array}\right)(-1)^{n}}{1+n z}=\frac{N ! z^{N}}{\prod_{n=0}^{N}(1+n z)} .
$$

Let $A(z)=\sum_{n=0}^{N} \frac{\left(\begin{array}{l}N \\ n\end{array}\right)(-1)^{N}}{1+n z}, B(z)=\prod_{n=0}^{N}(1+n z)$, and $G(z)=A(z) \cdot B(z)$. The order of $G(z)$ is $N$, thus it can be written as $G(z)=\sum_{i=0}^{N} g_{i} z^{i}$, where $g_{i}=\left.\frac{1}{i !} \frac{\partial^{i} G(z)}{\partial z^{i}}\right|_{z=0}$. It can be easily shown that

$$
\frac{\partial^{i} A(z)}{\partial z^{i}}=(-1)^{i} i ! \sum_{n=0}^{N} \frac{\left(\begin{array}{c}
N \\
n
\end{array}\right)(-1)^{n} n^{i}}{(1+n z)^{i+1}} .
$$

Using the identity obtained in [[18], $(0.154,3-4)]$ as

$$
\sum_{n=0}^{N}\left(\begin{array}{l}
N \\
n
\end{array}\right)(-1)^{n} n^{i}=\left\{\begin{array}{ll}
0, & 0 \leq i<N \\
(-1)^{N} N !, & i=N
\end{array},\right.
$$


we get

$$
\begin{aligned}
\left.\frac{\partial^{i} A(z)}{\partial z^{i}}\right|_{z=0} & =(-1)^{i} i !\left(\sum_{n=0}^{N}\left(\begin{array}{l}
N \\
n
\end{array}\right)(-1)^{n} n^{i}\right) \\
& =\left\{\begin{array}{ll}
0, & 0 \leq i<N \\
(N !)^{2}, & i=N
\end{array} .\right.
\end{aligned}
$$

Since, $\left.A(z)\right|_{z=0}=0$ and $\left.B(z)\right|_{z=0}=1$, thus

$$
\begin{aligned}
g_{i} & =\left.\frac{1}{i !} \frac{\partial^{i}(A(z) \cdot B(z))}{\partial z^{i}}\right|_{z=0}=\left.\left.\frac{1}{i !} \frac{\partial^{i} A(z)}{\partial z^{i}}\right|_{z=0} \cdot B(z)\right|_{z=0} \\
& =\left\{\begin{array}{ll}
0, & 0 \leq i<N \\
N !, & i=N
\end{array} .\right.
\end{aligned}
$$

Thus,

$$
A(z)=\frac{G(z)}{B(z)}=\frac{N ! z^{N}}{\prod_{n=0}^{N}(1+n z)},
$$

which proves (54). Replacing $z=\frac{y}{x}$ in (54), we obtain Lemma 1 and the proof is complete.

\section{REFERENCES}

[1] K. J. R. Liu, A. K. Sadek, W. Su, and A. Kwasinski, Cooperative Communications and Networking. Cambridge University Press, 2008.

[2] J. N. Laneman, D. N. C. Tse, and G. W. Wornell, "Cooperative diversity in wireless networks: efficient protocols and outage behaviour," IEEE Trans. Inform. Theory, vol. 50, pp. 3062-3080, Dec. 2004.

[3] J. N. Laneman and G. W. Wornell, "Distributed space-time coded protocols for exploiting cooperative diversity in wireless networks," IEEE Trans. Inform. Theory, vol. 49, pp. 2415-2525, Oct. 2003.

[4] W. Su, A. K. Sadek, and K. J. R. Liu, "SER performance analysis and optimum power allocation for decode-and-forward cooperation protocol in wireless networks," in Proc. IEEE Wireless Communications and Networking Conference (WCNC'05), vol. 2, pp. 984-989, Mar. 2005.

[5] W. Su, A. K. Sadek, and K. J. R. Liu, "Cooperative communication protocols in wireless networks: performance analysis and optimum power allocation," Wireless Personal Commun., vol. 44, pp. 181-217, Jan. 2008.

[6] A. K. Sadek, W. Su, and K. J. R. Liu, "Multi-node cooperative communications in wireless networks," IEEE Trans. Signal Processing, vol. 55, no. 1, pp. 341-355, Jan. 2007.

[7] B. Zhao and M. C. Valenti, "Practical relay networks: A generalization of hybrid-ARQ," IEEE J. Select. Areas Commun., vol. 23, no. 1, pp. 7-18, Jan. 2005.

[8] M. Zorzi and R. R. Rao, "Geographic random forwarding (GeRaF) for ad hoc and sensor networks: multihop performance," IEEE Trans. Mobile Comput., vol. 2, no. 4, pp. 337-348, Oct.Dec. 2003.

[9] M. Zorzi and R. R. Rao, "Geographic random forwarding (GeRaF) for ad hoc and sensor networks: energy and latency performance," IEEE Trans. Mobile Comput., vol. 2, no. 4, pp. 349-365, Oct.Dec. 2003.

[10] J. Luo, R. S. Blum, L. J. Greenstein, L. J. Cimini, and A. M. Haimovich, "New approaches for cooperative use of multiple antennas in ad hoc wireless networks," in Proc. IEEE 60th Vehicular Technology Conference, vol. 4, pp. 2769-2773, Sept. 2004.

[11] A. S. Ibrahim, A. K. Sadek, W. Su, and K. J. R. Liu, "Cooperative communications with partial channel state information: when to cooperate?" in Proc. IEEE Global Telecommunications Conference (Globecom'05), pp. 3068-3072, vol. 5, Nov. 2005.

[12] A. S. Ibrahim, A. K. Sadek, W. Su, and K. J. R. Liu, "Relay selection in multi-node cooperative communications: when to cooperate and whom to cooperate with?" in Proc. IEEE Global Telecommunications Conference (Globecom'06), pp. 1-5, Nov. 2006.

[13] D. G. Brennan, "Linear diversity combining techniques," Proc. IEEE, vol. 91, no. 2, pp. 331-356, Feb. 2003.

[14] M. O. Hasna and M. -S. Alouini, "Performance analysis of two-hop relayed transmssions over rayleigh fading channels," in Proc. IEEE Vehicular Technology Conference, vol. 4, pp. 1992-1996, Sepy. 2002.

[15] J. G. Proakis, Digital communications, 4th ed. McGraw-Hill, 2000.

[16] M. Abramowitz and I. A. Stegun, Handbook of Mathematical Functions with Formulas, Graphs, and Mathematical Tables. New York: Dover publications, 9th ed., 1970.
[17] M. K. Simon and M.-S. Alouini, "A unified approach to the performance analysis of digital communication over generalized fading channels," Proc. IEEE, vol. 86, no. 9, pp. 1860-1877, Sept. 1998.

[18] I. S. Gradshteyn and I. M. Ryzhik, Tables of Integrals, Series, and Products. San Diego, CA: Academic press, 5th ed., 1994.

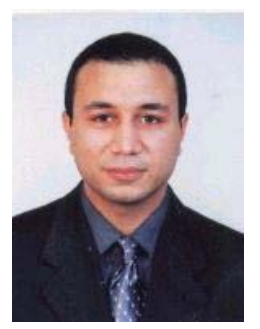

Ahmed S. Ibrahim (S'04) received the B.S. (with highest honors) and M.S. degrees in electrical engineering from Cairo University, Cairo, Egypt, in 2002 and 2004, respectively, and he is currently pursuing his Ph.D. degree at the Electrical and Computer Engineering Department, University of Maryland, College Park.

His research interests include cooperative communications and networking, cross-layer design of wireless networks, MIMO-OFDM systems, and signal processing algorithms for data manipulation.

Mr. Ibrahim is a recipient of the Graduate School Fellowship from the University of Maryland in 2004 and 2005.

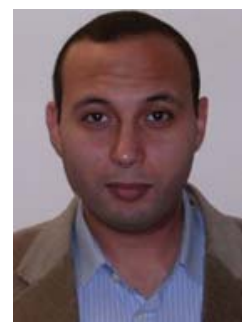

Ahmed K. Sadek (M'07) received the B.Sc. degree (with highest Honors) and the M.Sc. degree in electrical engineering from Alexandria University, Alexandria, Egypt in 2000 and 2003, respectively. $\mathrm{He}$ received the Ph.D. degree in electrical engineering from the University of Maryland, College Park, in 2007. He is currently a Senior Engineer with Corporate Research and Development division, Qualcomm Incorporated. His current research interests are in the areas of cognitive radios, spectrum sharing, cooperative communications, and MIMO-

OFDM systems.

In 2000, Dr. Sadek won the first prize in IEEE Egypt Section undergraduate student contest for his B.Sc. graduation project. He received the Graduate School Fellowship from the University of Maryland in 2003 and 2004, and the Distinguished Dissertation Fellowship award from the Department of Electrical Engineering, University of Maryland in 2007. He is a co-author of Cooperative Communications and Networking, Cambridge University Press, 2008.

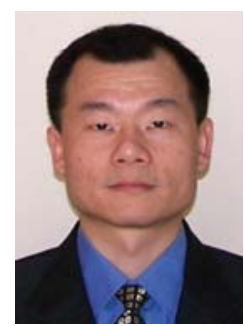

Weifeng Su (M'03) received the Ph.D. degree in electrical engineering from the University of Delaware, Newark in 2002. He received his B.S. and $\mathrm{Ph} . \mathrm{D}$. degrees in applied mathematics from Nankai University, Tianjin, China, in 1994 and 1999, respectively. His research interests span a broad range of areas from signal processing to wireless communications and networking, including spacetime coding and modulation for MIMO wireless communications, MIMO-OFDM systems, cooperative communications for wireless networks, and ultra-wideband (UWB) communications.

Dr. Su has been an Assistant Professor at the Department of Electrical Engineering, State University of New York (SUNY) at Buffalo since March 2005. From June 2002 to March 2005, he was a Postdoctoral Research Associate with the Department of Electrical and Computer Engineering and the Institute for Systems Research (ISR), University of Maryland, College Park. Dr. Su received the Signal Processing and Communications Faculty Award from the University of Delaware in 2002 as an outstanding graduate student in the field of signal processing and communications. In 2005, he received the Invention of the Year Award from the University of Maryland. Dr. Su has been an Associate Editor of IEEE Transactions on Vehicular Technology, IEEE Signal Processing Letters, and a Guest Editor of special issue on cooperative communications and networking of IEEE Journal on Selected Areas in Communications. 


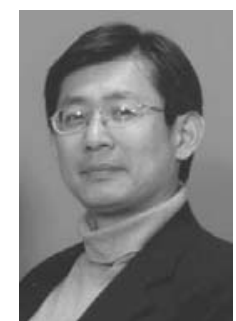

K. J. Ray Liu (F'03) received the B.S. degree from the National Taiwan University and the Ph.D. degree from UCLA, both in electrical engineering. $\mathrm{He}$ is Professor and Associate Chair, Graduate Studies and Research, and Director of Communications and Signal Processing Laboratory of Electrical and Computer Engineering Department, University of Maryland, College Park. He leads the Maryland Signals and Information Group conducting research encompassing broad aspects of information technology including signal processing, communications, networking, information forensics and security, biomedical and bioinformatics.

Dr. Liu is the recipient of numerous honors and awards including best paper awards from IEEE Signal Processing Society (twice), IEEE Vehicular Technology Society, and EURASIP; IEEE Signal Processing Society Distinguished Lecturer, EURASIP Meritorious Service Award, and National Science
Foundation Young Investigator Award. He also received various teaching and research recognitions from University of Maryland including universitylevel Distinguished Scholar-Teacher Award, Invention of the Year Award, and college-level Poole and Kent Company Senior Faculty Teaching Award.

Dr. Liu is Vice President - Publications and on the Board of Governor of IEEE Signal Processing Society. He was the Editor-in-Chief of IEEE Signal Processing Magazine and the founding Editor-in-Chief of EURASIP Journal on Applied Signal Processing.

His recent books include Cooperative Communications and Networking, Cambridge University Press, 2008; Resource Allocation for Wireless Networks: Basics, Techniques, and Applications, Cambridge University Press, 2008; Ultra-Wideband Communication Systems: The Multiband OFDM Approach, IEEE-Wiley, 2007; Network-Aware Security for Group Communications, Springer, 2007; Multimedia Fingerprinting Forensics for Traitor Tracing, Hindawi, 2005. 